\title{
Sexual Dimorphisms in Spatial Memory, Facial Recognition, and Reaction Time
}

\author{
Hannah R. Waller ${ }^{a} \&$ Anthony L. Lin ${ }^{b}$
}

\begin{abstract}
Objectives: Literature suggests that there is a female advantage in facial recognition, and a male one in spatial memory. Researchers in this study have developed a new measure for assessing these constructs, which could also provide insight into the cognition of an increasingly elderly population. Researchers investigated whether females outperformed males on a Novel Faces and Places (NFAP) test, which uses facial recognition and spatial memory measures, as well as inherently monitoring reaction times. NFAP was adapted from a similar test, which uses objects instead of faces. Methods: College students (N=90) completed a demographics survey and took NFAP at one of 5 different display duration conditions. Results: No significant differences were found among the sexes for total NFAP score or for Novel Faces or Novel Places identification. However, females had faster reaction times overall, and particularly when making correct judgments. Female correct reaction time was significantly faster than female incorrect reaction time and than both male correct and incorrect reaction times. Also observed was a display duration effect, where a 15 second display duration yielded the highest scores, followed by 12 seconds, compared to 9 , 6 , or 3 seconds. Conclusion: These findings indicate that males and females process visual information in different ways. Though no significant differences in score were found, the female advantage seen in other tests of spatial memory was not observed on NFAP as was hypothesized.
\end{abstract}

Keywords: Sexual dimorphism, memory, reaction time

A rapidly expanding literature base has lead researchers to propose that there exist distinct sexual dimorphisms in various neurocognitive functions, particularly memory. For instance, studies have found that women outperform men on tests of episodic (Herlitz, Nilsson, \& Baekman, 1997), emotional, (Canli, Desmond, Zhao, \& Gabrieli, 2001) and verbal memory (Bleecker, Bolla-Wilson, Agnew, \& Meyers, 2006). Additionally, the neuropsychological Novel Image Novel Location (NINL) task has been used as a test of spatial memory capacity in young adult populations and has found female advantages in this construct as well (Piper, Yasen, \& Miller, 2011). The examination of sexual dimorphisms in performance when faces are used in place of objects in NINL is an area that has not yet been explored.

Though few studies have examined spatial memory in conjunction with facial recognition, Chen and McNamara (2011) conducted two separate experiments where participants memorized the location of either human or non-human animal avatars and researchers found that participants performed much better when remembering the location (and orientation) of the humans. The authors suggest that perhaps there is something about an implied social interaction that aids encoding of memories. It is also possible that animal avatars are processed more similarly to objects than human avatars are, and thus are not remembered as well. These results could have important implications because whereas NINL uses objects (Piper et al., 2011a), the substituted use of faces in a similar test should prompt better spatial memory and serve as a valid means of measuring it.

Contrary to the female advantage found in the Piper et al. (2011b) study, Astur, Oritz, and
Sutherland (1998) found that males greatly outperformed females on a virtual Morris water maze test, which, like NINL, was used to measure spatial memory. Perhaps the data from the Piper et al. (2011b) study opposed that of Astur et al. because objects can be verbally labeled in NINL, and research confirms that women are consistently superior in verbal memory (Herlitz et al., 1997). A task that uses faces instead of objects and that also measures spatial memory will further test whether verbal labeling accounts for the female advantage. Since faces are not as easy to verbally label as are objects, isolation of spatial memory from verbal memory is more likely in such a task, and the female advantage should disappear.

Consistent with the verbal advantage found in females and the spatial one in males, Lewin, Wolgers, and Herlitz (2001) examined verbal and visuospatial memory and found that women outperformed men on verbal episodic memory tasks, whereas men's performance was superior for tasks requiring visuospatial memory. If these results are replicated, men should perform better than women on a test measuring spatial memory and facial recognition, as verbal labeling of faces is more difficult than for objects and because the task requires recruitment of visuospatial memory. Furthermore, because there is limited literature on sexual dimorphisms regarding facial recognition, it remains unclear whether this component will favor one sex or the other.

One study that did observe sexual dimorphisms in facial recognition was that of Lewin and Herlitz (2002). The researchers gave participants a facial recognition task whereby they were shown a 
series of faces for varying durations depending on condition and were later shown another series of faces, where some were images they had seen before and others were "distracters." Participants were then asked to state whether they had seen each face previously. Results indicated that females outperformed males, but only in recognition of other female faces. Given the findings of Astur et al. (1998) and Lewin et al. (2001), it is expected that since men generally outperform women in tasks of spatial memory, when this element is added to the facial memory task, the female advantage will no longer be observed.

In order to carry out this experiment, the researchers have created a novel instrument designed to examine spatial memory and facial recognition, and inherently, reaction time. Although sex differences in ability to identify novel faces and novel places are the primary focus of this paper, differences in reaction time are also of interest. Existing literature allows researchers to suggest that males consistently have faster reaction times, particularly in response to visual stimuli. Dimorphisms in reaction time between the sexes are compelling because they suggest possible differences in the neural organization and processing techniques used in males and females. When reaction times were recorded and brain activity monitored with fMRI, differential patterns of activation were observed on several tests of visual working memory where males displayed bilateral activation of the lateral prefrontal cortices, parietal cortices, and caudate nucleus, whereas females showed activity predominantly in the left hemisphere in these same structures. Additionally, females were found to be more accurate with slower reaction times while males made their decisions more quickly, but less accurately (Speck et al., 2000). These findings suggest not only that there exist differences in functional brain organization between males and females, but also that this organization results in the recruitment of separate processing strategies when working memory is activated. If the novel instrument used in the present study can detect these distinct processing strategies, it may hold promise for use in other populations such as the elderly or cognitively impaired.

In support of the hypothesis that males and females utilize sexually dimorphic processing strategies are the findings from a study in which females and males each completed a mental rotation task of 3-D objects while brain activity was monitored with ERP and reaction times recorded. While this study did not find significant differences in reaction times between the males and females, neuronal activation patterns were revealed predominantly in parietal regions in males whereas females showed activation in inferior frontal regions in addition (Hugdahl, Thomsen, \& Ersland, 2006). The authors propose that the sexes process visuo-spatial information differently, with males taking a concurrent, global approach and females a more sequential one.

Apart from differences in performance based on sex, effects of encoding time, collapsing across sex, are also of interest. Hirschman and
Hostetter (2000) demonstrated in a visual, orthographic memory task that as display duration of stimuli increases, accuracy of memory for those stimuli improves. However, in this task spatial location of stimuli was not manipulated, so a task that measures spatial memory at varying display durations could provide further insight into the nature of visual memory.

To measure the effects of encoding time upon memory and to test the hypothesis that the female advantage observed NINL will no longer be present in a new test that uses faces rather than objects to measure spatial memory, the current study uses the Novel Faces and Places (NFAP) test adapted from NINL. This study will expand upon current knowledge of sex differences in various neurocognitive tasks in terms of spatial memory, facial recognition and reaction time.

\section{Methods}

Participants

This study recruited 34 male and 56 female students from introductory Psychology classes. Students received course credit for their participation. All participants were between the ages of 18 and 22 .

\section{Procedures}

All procedures were submitted to and approved by the Willamette University IRB. All participants signed informed consent forms and filled out a short demographics survey (Appendix A).

\section{Assessments and Measures}

Participants completed a brief questionnaire inquiring about their age, gender, and handedness.

NFAP was adapted from a previous version of NINL, with the major differences being the use of facial images rather than objects. Like NINL, each of 24 frames in NFAP consisted of four quadrants with images in three of the four where the empty quadrant changed location with each new frame. Images were obtained from an online mug shot database and were edited to suit the requirements of the program. The images were $50 \%$ female, $50 \%$ male and $70 \%$ white, $30 \%$ non-white. Care was also taken to ensure that images selected did not have any distinctive features such as bruises, definitive emotional expressions or facial piercings and that age distribution among the faces used was even (Tables 1 and 2).

\section{Procedures}

Participants were instructed to memorize each face and in which quadrant of the frame it was located as part of the "learn" phase. Each of the frames was shown for a fixed amount of time, either (a) 3 seconds, (b) 6 seconds, (c) 9 seconds, (d) 12 seconds, or (e) 15 seconds. Immediately after the learn phase, testing began. During the test phase participants were asked to identify, on each of the frames, whether one of the faces had changed location, whether a novel 
face was present, or whether there was no change from the learned frame. The test was scored such that zero points were given when participants failed to recognize when a change had occurred, one point for identifying that a change had occurred, two points for correctly identifying the change type (novel face/novel place) and three points for identifying correct change type and in which quadrant the change occurred. A correct no change identification was also awarded three points. Response times were measured and stimuli were administered using the Psychology Experiment Building Language software (Mueller, 2005), version 0.11. Participants were tested 5 at a time, and were allowed to leave when finished, regardless of the progress of their peers.

\section{Data Analysis}

Statistical analyses were performed using SYSTAT, version 13.0 (Systat Software, 2008) with an alpha level of .05. Two-sample t-tests were performed for sex and each change category individually (Novel Face (NF), Novel Place (NP), or No Change (NC) and for the total NFAP score (NF + $\mathrm{NP}+\mathrm{NC}$ ). Two-sample t-tests were also run for encoding times (two at a time) and total correct NFAP score. A paired t-test was run between sex and reaction time for each category and for overall score. Pearson product moment correlations were completed for reaction times on NF, NP, and NC categories.

Table 1: FAP Image Characteristics by Frame, Learn Phase (Note. Table 1 displays the characteristics of each image shown in the learn phase where the numbers on the left are the 24 frames and where "NW, NE, SW and SE" are the four quadrants of the frame beginning in the upper left hand corner and moving clockwise. A “-1" indicates a blank quadrant)

\begin{tabular}{|c|c|c|c|c|c|c|c|c|}
\hline 1 & $\begin{array}{l}\text { NW_ethnicty } \\
\mathrm{x}\end{array}$ & $\begin{array}{l}\text { NE_ethnicity } \\
\text { whte }\end{array}$ & $\begin{array}{l}\text { SW_ethnicty } \\
\text { whte }\end{array}$ & $\begin{array}{l}\text { SE_et hnicity } \\
\text { nonwhte }\end{array}$ & MW_Sex & $\begin{array}{l}\text { quesex } \\
\text { fe_semab }\end{array}$ & $\begin{array}{l}\text { SW_Sex } \\
\text { mab }\end{array}$ & $\begin{array}{l}\text { SE_Sex } \\
\text { femat }\end{array}$ \\
\hline 2 & normwhte & whte & $\mathrm{x}$ & whte & mab & femab & $\mathrm{x}$ & $\mathrm{mab}$ \\
\hline $\begin{array}{l}3 \\
4\end{array}$ & $\begin{array}{l}\text { whte } \\
\text { whte }\end{array}$ & $\begin{array}{l}\text { nonwhte } \\
\text { whte }\end{array}$ & $\begin{array}{l}\text { whte } \\
x\end{array}$ & $\begin{array}{l}x \\
\text { nonwhte }\end{array}$ & $\begin{array}{l}\text { fermab } \\
\text { mab }\end{array}$ & $\begin{array}{l}\text { femab } \\
\mathrm{mab}\end{array}$ & ${ }_{x}^{m a k}$ & $\begin{array}{l}x \\
\text { femab }\end{array}$ \\
\hline 5 & normuhte & $\mathrm{x}$ & whte & whte & $\mathrm{mab}$ & $\mathrm{x}$ & fermab & femab \\
\hline 6 & whte & nonwhte & $\mathrm{x}$ & whte & $\mathrm{mab}$ & femab & $\mathrm{x}$ & $\mathrm{mab}$ \\
\hline 7 & whte & $x$ & nonwhte & whte & fermab & $x$ & fermab & $\mathrm{mab}$ \\
\hline 8 & & whte & whte & nonwhte & $x$ & mab & mab & femab \\
\hline 9 & norwhte & $\mathrm{x}$ & whte & whte & $\mathrm{mab}$ & $\mathrm{x}$ & fermab & femak \\
\hline 10 & $x$ & nonwhte & whte & whte & $x$ & mab & mak & femak \\
\hline 11 & whte & nonwhte & whte & $x$ & fermab & mab & femak & $x$ \\
\hline 12 & norwhte & whte & $\mathrm{x}$ & whte & $\mathrm{mab}$ & mab & $\mathrm{x}$ & fermak \\
\hline 13 & norwhte & whte & whte & $x$ & fente & femab & mab & $\mathrm{x}$ \\
\hline 14 & $x$ & whte & whte & nonwhte & $x$ & mab & femab & mab \\
\hline 15 & norwhte & whte & whte & $\mathrm{x}$ & femab & femab & mab & $\mathrm{x}$ \\
\hline 16 & whte & & whte & nonwhte & mab & $x$ & $\mathrm{mak}$ & femab \\
\hline 17 & whte & nonwhte & whte & $x$ & femab & mab & femal & \\
\hline 18 & $x$ & whte & nonwhte & whte & $x$ & mab & mak & fermab \\
\hline 19 & whte & whte & norwhte & $\mathrm{x}$ & femae & femab & mab & $\mathrm{x}$ \\
\hline 20 & $\mathrm{x}$ & nonwhte & whte & whte & $x$ & femab & $\mathrm{mab}$ & fermab \\
\hline 21 & whte & & nonwhte & whte & $\mathrm{mak}$ & & femak & $\mathrm{mab}$ \\
\hline 22 & whte & whte & $x$ & whte & fermab & femab & $x$ & $\mathrm{mab}$ \\
\hline 23 & whte & whte & $\mathrm{x}$ & whte & mak & femab & $\hat{x}$ & mab \\
\hline 24 & $x$ & whte & whte & whte & $x$ & femab & femak & mab \\
\hline
\end{tabular}

\begin{tabular}{|c|c|c|}
\hline SE_Sex & MW_age & NE_age \\
\hline & -1 & \\
\hline ab & 19 & \\
\hline & 46 & \\
\hline rab & 19 & \\
\hline emak & 32 & \\
\hline rab & 21 & \\
\hline & 46 & \\
\hline emak & -1 & \\
\hline ab & 24 & \\
\hline female & -1 & \\
\hline k & 30 & \\
\hline emak & 23 & \\
\hline & 30 & \\
\hline nab & -1 & \\
\hline k & 32 & \\
\hline pab & 18 & \\
\hline & 21 & \\
\hline emak & -1 & \\
\hline & 46 & \\
\hline & -1 & \\
\hline & 24 & \\
\hline & 51 & \\
\hline & 36 & \\
\hline & -1 & \\
\hline
\end{tabular}

\begin{tabular}{|c|c|}
\hline & SE_age \\
\hline 36 & 25 \\
\hline 21 & -1 \\
\hline 22 & 34 \\
\hline 34 & -1 \\
\hline-1 & 23 \\
\hline 27 & -1 \\
\hline-1 & 28 \\
\hline 43 & 26 \\
\hline-1 & 35 \\
\hline 26 & 46 \\
\hline 27 & 28 \\
\hline 28 & -1 \\
\hline 35 & 29 \\
\hline 30 & 20 \\
\hline 24 & 31 \\
\hline-1 & 21 \\
\hline 43 & 38 \\
\hline 18 & 41 \\
\hline 21 & 42 \\
\hline 32 & 42 \\
\hline-1 & 35 \\
\hline 29 & -1 \\
\hline 56 & -1 \\
\hline 37 & 38 \\
\hline
\end{tabular}

Table 2: NFAP Image Characteristics by Frame, Test Phase

(Note. Table 2 displays characteristics of each image shown in the test phase where the numbers on the left are the 24 frames and where "NW, NE, $S W$ and $S E$ " are the four quadrants of the frame beginning in the upper left hand corner and moving clockwise. A “-1" indicates a blank quadrant)

\begin{tabular}{|c|c|c|c|c|}
\hline 1 & $\begin{array}{l}\text { MW_ethnity } \\
\text { whte }\end{array}$ & $\begin{array}{l}\text { NE_ethnicity } \\
\text { nonwhte }\end{array}$ & $\begin{array}{l}\text { SW_et hnicty } \\
x\end{array}$ & $\begin{array}{l}\text { SE_et hnicity } \\
\text { whte }\end{array}$ \\
\hline 2 & whte & $x$ & whte & nonwhte \\
\hline $\begin{array}{l}3 \\
4\end{array}$ & $\begin{array}{l}\text { whte } \\
\text { nonwhte }\end{array}$ & $\begin{array}{l}\text { whte } \\
\text { nonwhte }\end{array}$ & $\begin{array}{l}x \\
\text { whte }\end{array}$ & $\begin{array}{l}\text { whte } \\
x\end{array}$ \\
\hline 5 & & nonwhte & whte & whte \\
\hline 6 & whte & $x$ & whte & whte \\
\hline $\begin{array}{l}7 \\
8\end{array}$ & $\begin{array}{l}\text { nonwhte } \\
\text { whte }\end{array}$ & $\begin{array}{l}x \\
\text { nonwhte }\end{array}$ & $\begin{array}{l}\text { whte } \\
\text { nonwhte }\end{array}$ & $\begin{array}{l}\text { whte } \\
x\end{array}$ \\
\hline 9 & & whte & nonwhte & whte \\
\hline 10 & nonwhte & $\mathrm{x}$ & whte & whte \\
\hline 11 & $x$ & whte & whte & nonwhte \\
\hline 12 & whte & nonwhte & $x$ & whte \\
\hline 13 & whte & nonwhte & $x$ & whte \\
\hline 14 & $\mathrm{x}$ & whte & whte & nonwhte \\
\hline 15 & whte & whte & nonwhte & $\mathrm{x}$ \\
\hline 16 & whte & $\mathrm{x}$ & whte & whte \\
\hline 17 & whte & whte & $x$ & whte \\
\hline 18 & whte & whte & $x$ & whte \\
\hline 19 & nonwhte & whte & whte & $\mathrm{x}$ \\
\hline 20 & nonwhte & $x$ & whte & whte \\
\hline 21 & whte & $\mathrm{x}$ & nonwhte & whte \\
\hline 22 & nonwhte & whte & whte & $\mathrm{x}$ \\
\hline 23 & $\mathrm{x}$ & nonwhte & whte & nonwhte \\
\hline 24 & nonwhte & whte & whte & $\mathrm{x}$ \\
\hline
\end{tabular}

\section{Results}

Among the seven conditions, participants did not differ based on sex, age or handedness. Two-

$\begin{array}{ll}\text { SW_Sex } & \text { SE_Sex } \\ x & m \\ m & f \\ x & f \\ f & x \\ m & f \\ f & m \\ f & f \\ \text { m } & x \\ \text { m } & f \\ \text { f } & m \\ m & f \\ x & m \\ x & m \\ m & f \\ m & x \\ f & m \\ x & m \\ x & m \\ m & x \\ m & f \\ f & m \\ f & x \\ \text { f } & m \\ m & x\end{array}$

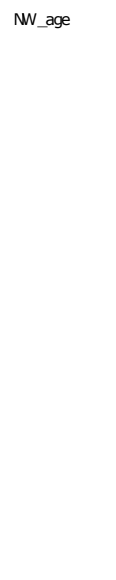

sample t-tests were run to determine sexual dimorphisms in performance both for the overall score and for each change type individually. Though 
females did not differ from males in overall correct score $(\mathrm{t}(90)=0.84, \mathrm{p}=0.40)$ or in correct responses to $\mathrm{NC}(\mathrm{t}(90)=1.77, \mathrm{p}=0.80), \mathrm{NF}(\mathrm{t}(90)=-.59, \mathrm{p}=$ $0.56)$, or NP $(\mathrm{t}(90)=0.97, \mathrm{p}=0.94)$ change types, females did have significantly faster overall reaction times than males, collapsing across all seven encoding times. Also of interest is the significantly faster reaction time in females when making correct responses versus when making incorrect responses (Figure 1). Overall mean reaction times were significantly different between males and females as well $(p=.035)$. Additionally, moderate correlations between reaction times for novel face and no change, between novel face and novel place, and between novel place and no change, collapsing across sex (Figures 2, 3, and 4) were observed.

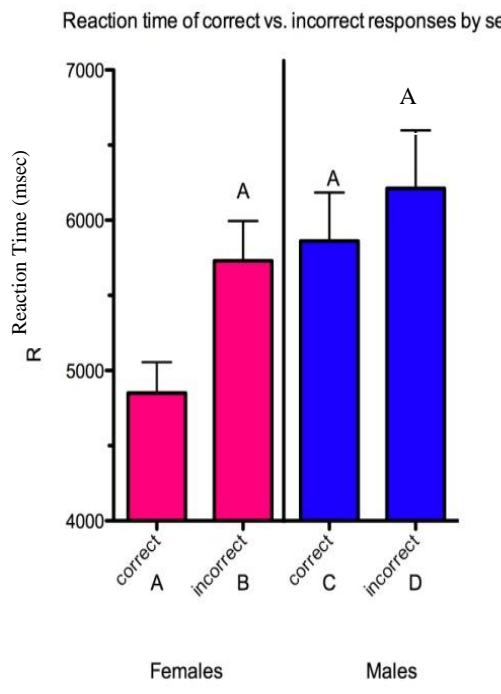

Figure 1: Median total reaction times for both females (left) and males (right) in responses that were either correct or incorrect. The letters above the bars indicate with which other bars there was a significant difference.

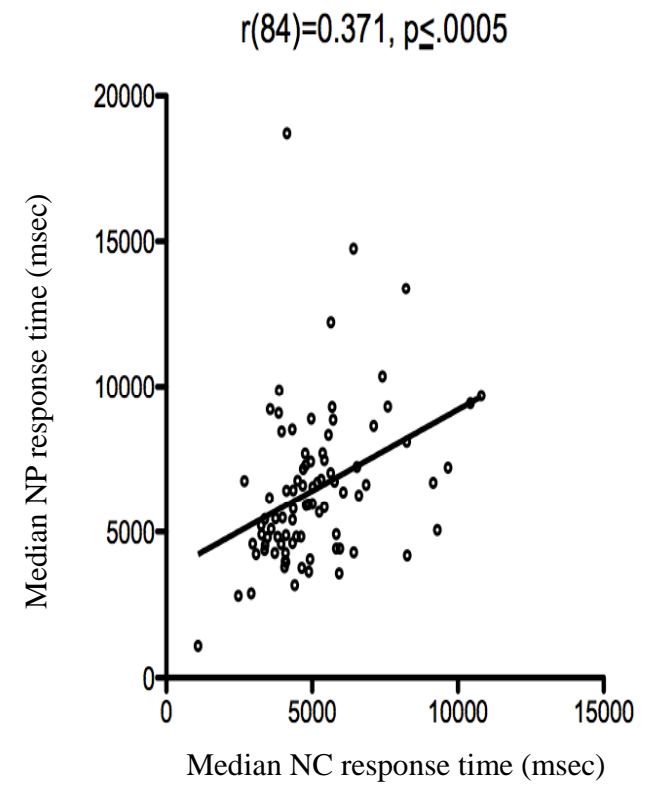

Figure 2: Correlation between reaction time for NP versus NC responses $\mathrm{r}(84)=0.371, \mathrm{p} \leq .0005$. 


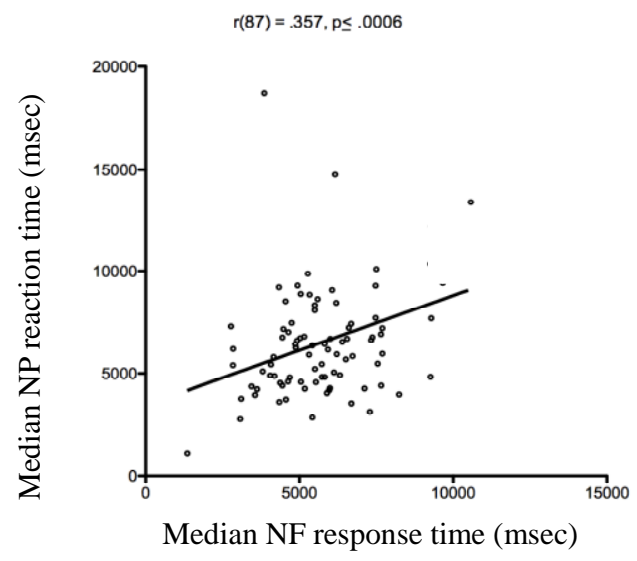

Figure 3: Correlation between reaction time for NP versus NF responses $r(87)=.357, \mathrm{p} \leq .0006$.

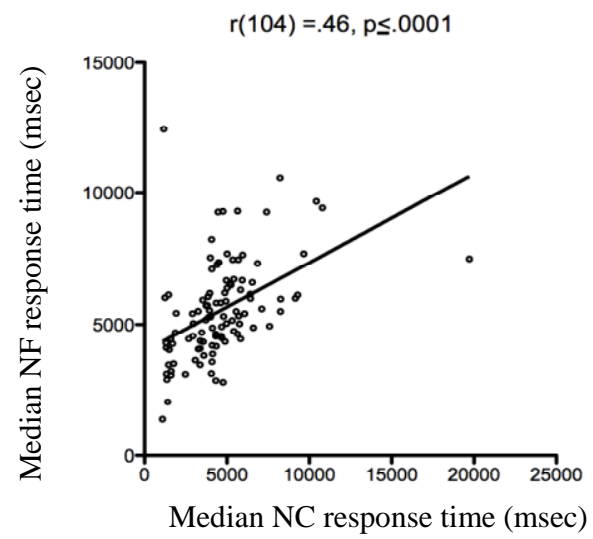

Figure 4: Correlation between reaction time for NF versus NC responses $r(87)=.46, p \leq .0001$.

The five different display durations tested also produced varying results in terms of overall NFAP score (Figure 5). Longer encoding times, particularly those above 9 seconds, resulted in significantly higher overall scores on NFAP.

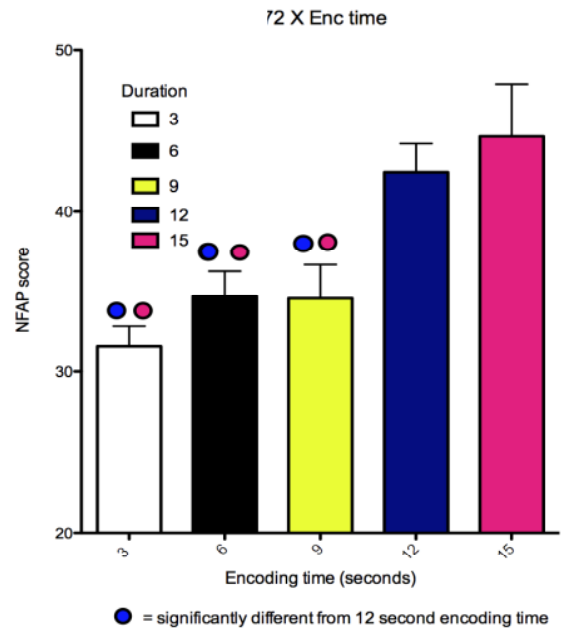

O = significantly different from 15 second encoding time

Figure 5: Total NFAP scores by encoding time 


\section{Discussion}

The present study contributes to the understanding of neurocognitive processing differences in females and males, especially in regards to the sex difference found in reaction time. Females were faster than males overall, collapsing across all conditions, though they were not necessarily more accurate. Perhaps most compelling is the finding that female reaction time when making correct responses was significantly faster than their reaction time when making incorrect responses, where this incorrect reaction time did not differ from male reaction time for either correct or incorrect responses. This may imply that when females are confident that a change has occurred, either in novel place or face, they are much faster in identifying it as such. On the other hand, males did not differ in reaction time between correct and incorrect responses, which may indicate that they are overly confident in their incorrect responses, or perhaps that they were less sure all together than females, whether a frame in the test phase was different from the learned one.

Consistent with the hypothesis that men are falsely confident in incorrect answers more frequently than are women is the research of Lundeberg, Fox, and Puncochar (1994), which investigated sex differences in confidence judgments on course exams. While both sexes were found to be overconfident in both their correct and incorrect answers, men were significantly more overconfident when making an incorrect response. A remaining query then, is why this false confidence does not result in faster reaction times for men than for women. Future studies should address this issue.

Regardless of the direction of the reaction time sex effect, the existence of a difference at all is provocative in that it suggests differential methods of processing the same visual information. Consistent with this postulation are the findings from a study in which females and males completed a task of reaction time whereby they were required to give a verbal decision about stimuli that were placed in varying spatial locations. While differences in reaction time favoring males only approached significance, a crucial finding was that the sexes showed different patterns of reaction time improvement based on the location of the stimuli. Females exhibited a linear increase in reaction time only to objects in the left or right dimension, while males showed a step-like increase in reaction time to all stimuli, regardless of location (left/right, up/down) (Adam, 1999). This finding further supports the hypothesis that there are distinct differences in the ways the sexes process visual information, and could have important implications for NFAP, which utilizes a spatial manipulation component.

Findings from the Adam (1999) study may also indicate that men are less vulnerable to visuospatial manipulation and are able to focus more on the faces without being distracted by spatial changes in stimuli. Peculiarly though, if this is true, men should make faster and more accurate responses in NFAP. Since an opposing effect to this expected one was observed, future research should investigate whether men would excel on a visuo-spatial task similar to NFAP, but where participants have an unlimited amount of time to study the faces in the learn phase to understand whether the timed component is the reason males are not performing better than their female counterparts. Though the present study did not show significant sexual dimorphisms in overall score collapsing across display duration, the female advantage found in NINL (Piper, 2011b) was no longer observed, as was hypothesized. This effect could have been explained by the lack of verbal labeling ability inherent in NFAP, but present in NINL (Piper, 2011b).

In contemplating the potential cognitive processing differences between men and women in the context of NFAP, it is important to determine which aspect of the test they are encoding differently. Hugdahl et al. (2006) observed sexual dimorphisms in processing of spatial information unrelated to faces, Speck et al. (2000) isolated visual processing differences independent from the spatial component, and Lewin et al. (2002) found that males and females process faces in separate ways. Furthermore, Adam (1999) found that men and women have distinct reaction time patterns from one another in response to visual stimuli. These studies indicate that virtually every component of NFAP (visual, spatial, facial recognition, and reaction time) is being processed via modalities that are dependent upon sex. NFAP can be used as a means of gauging these differences concurrently, and subsequent studies should administer NFAP while simultaneously utilizing brain imaging technologies to identify the specific neural effects of such a test.

Researchers have found that tasks measuring reaction time are sensitive to age, where older participants have much slower reaction times (Fozard, Vercruyssen, Reynolds, Hancock, \& Quilter, 1993). An initial goal of the NFAP was to use it in conjunction with the Montreal Cognitive Assessment (MoCA) in elderly participants in efforts to determine the level of cognitive impairment at which facial recognition and spatial memory begin to decline. Due to time limitations and revisions to the test format and structure, this experiment was not able to be completed. Future research should examine the scores of elderly participants using NFAP at the 15-second display duration and should note both differences in performance based on level of cognitive impairment as well as sex differences that may exist in this population but that were not present in the young demographic.

The use of tests such as NFAP could be instrumental in measuring cognitive impairment in the elderly. Celone et al. (2006) recruited several elderly males and females and administered a basic blockdesign facial recognition task and the Clinical Dementia Rating Scale. The researchers found a moderate correlation between cognitive impairment 
and facial recognition. This suggests that tests of facial recognition could serve as a measure of detecting moderate cognitive impairment. Beyond this practical application is the significance of this correlation in quality of life of the elderly and their loved ones. Understanding the mental processes that are most vulnerable to dementia and other age-related cognitive impairment is crucial to effectively managing such ailments. Failure to recognize familiar faces can pose significant dangers to the elderly, especially when confusion occurs at the same time, which is often the reality in cases of dementia. Detecting loss of facial recognition in those with suspected cognitive impairment is an important procedure for ensuring the safety and well being of the elderly.

Though the use of NFAP holds promise for clinical applications, this study has some shortcomings. The only area where results were found to be significant was that of reaction time, a result that could be explained by the possible masking of sex differences by the use of several intervals in data analysis. Furthermore, a primary limitation to this study's methodology was a somewhat uneven number of male and female participants. This could have been due to a similar demographic in the Psychology classes, or perhaps more males chose to complete an alternate assignment than to participate in the study. Another limitation is that the rooms in which the data were collected were fairly small with the computers about 3 feet from each other. The close proximity of other participants may have made some people nervous or anxious. Additionally, the doors to the rooms were left open during testing so that participants could easily come in and out. The noise from others in the hallways as well as the distraction from participants entering and exiting the room may have negatively impacted scores.

Novel Faces and Places was run with five different display durations, primarily in efforts to determine whether the test was simply too difficult for participants and also to examine effects of encoding time on memory. The display times of 3 and 6 seconds were judged to be too difficult for participants as they received less than half of the possible points, and the 9 second condition was determined to still be slightly too difficult for many based on overall scores. The 12 and 15 second conditions were then tested and it was discovered that the 15-second display time showed the highest performance and thus the most effective encoding of memory.

The results from this research can be generalized to the broader population only to a limited extent. Our sample was from one narrow age group only and it is reasonable to suspect that the extrinsic motivation where students were required to participate as part of their course grades impacted their investment in the study and made them more apathetic with regards to their performance than would have been the case with a purely volunteer population. Additionally, if NFAP is used in elderly populations, new obstacles may present themselves such as dexterity and computer familiarity and experience. It is likely that many elderly individuals would have far less experience with technology than the college sample, so a computerized test may be somewhat challenging or frustrating for this population.

Consistent with previous findings, this study observed certain sexual dimorphisms in reaction time and processing strategy. When NINL was modified to remove the ability to verbally label images by utilization of faces in place of objects, the female advantage was no longer present. Furthermore, males and females did not differ in their overall scores nor did they differ in their scores on correct identification of each change type (NL, NF, or NC), but females did make their correct judgments faster than did male participants. Moreover, because reaction time and facial recognition have both been found to be sensitive to age, NFAP could be applied as a form of cognitive assessment in the elderly. Reaction time is important for daily tasks such as driving and danger avoidance, and facial recognition is crucial for ensuring safety and quality of life of the elderly. Testing for the deterioration of these fundamental cognitive functions can protect and prolong the lives of an increasingly aging population.

\section{Acknowledgements}

Special thanks are owed to Drs. Brian Piper, Susan Koger, and Jeremy Miller for their assistance in editing this manuscript and guidance in this experiment, and to Shelbie Wolfe for her assistance in data collection

\section{References}

Adam, J.J. (1999). Gender differences in choice reaction time: evidence for differential strategies. Ergonomics, 42, 327-332.

Astur, R.S., Ortiz, M.L., \& Sutherland R.J. (1998). A characterization of performance by men and women in a virtual Morris water task: A large and reliable sex difference. Behavioural Brain Research, 93, 185-190.

Bleecker M.L., Bolla-Wilson, K., Agnew, J., \& Meyers, D.A. (2006). Age-related sex differences in verbal memory. Journal of Clinical Psychology, 44(3), 403-411.

Canli, T., Desmond, J.E., Zhao, Z., \& Gabrieli, J.D.E. (2001). Sex differences in the neural basis of emotional memories. Proceedings of the National Academy of Sciences of the United States of America, 99 (16). Retrieved from: http://www.pnas.org/content/99/16/10789.fu ll.pdf $+\mathrm{html}$.

Celone, K.A., Calhoun, V.D., Dickerson, B.C., Atri, A., Chua, E.F., Miller, S.L., DePeau, K., Rentz, D.M., Selkoe, D.J., Blacker, D., Albert, M.S., \& Sperling, R.A. (2006). Alterations in memory networks in Mild Cognitive Impairment and Alzheimer's Disease: An independent component analysis. Journal of Neuroscience, 26, 10222-10231. 
Chen, X., \& McNamara, T. (2011). Object-centered reference systems and human spatial memory. Psychonomic Bulletin and Review, 18, 985991.

Fozard, J.L., Vercruyssen, M., Reynolds, S.L., Hancock, P.A., \& Quilter, R.E. (1994). Age Differences and Changes in Reaction Time: The Baltimore Longitudinal Study of Aging. Journal of Gerontology, 49, 179-189.

Herlitz, A., Nilsson, L.G., \& Baeckman, L. (1997). Gender differences in episodic memory. Memory and Cognition, 25 (6), 801-811.

Hirshman, E., \& Hostetter, M. (2000). Using ROC curves to test models of recognition memory: The relationship between presentation duration and slope. Memory \& Cognition, 28, 6-66.

Hugdahl, K., Thomsen, T., \& Ersland, L. (2006). Sex differences in visuo-spatial processing: An fMRI study of mental rotation. Neuropsychologia, 44, 1575-1583.

Lewin, C., Herlitz, A., \& Wolgers, G. (2001). Sex differences favoring women in Verbal But Not in Visuospatial Episodic Memory. Neuropsychology, 15(2), 165-173.

Lewin, C. \& Herlitz, A. (2002). Sex differences in face recognition-Women's faces make the difference. Brain and Cognition, 50, 121-128.

Lundeberg, M. A., Fox, P. W., \& Puncochaf, J.
(1994). Highly confident but wrong: Gender differences and similarities in confidence judgments. Journal of Educational Psychology, 86, 114-121.

Mueller, Shane (2005). Psychology Experiment Building Language Software (Version 0.11) [software]. Retrieved from http://pebl.sourceforge.net/.

Piper, B. J., Acevedo, S. F., Edwards, K. R., Curtiss, A. B., McGinnis, G. J., \& Raber, J. (2011a). Age, sex, and handedness differentially contribute to neurospatial function on the Memory Island and Novel-Image NovelLocation tests. Physiology \& Behavior, 103(5), 513-522.

Piper, B.J., Yasen, A.L., \& Miller, J.K. (2011b). Examination of sexually dimorphic behavior on the Novel-Image Novel-Location recognition memory test. Journal of Behavioral and Brain Science, 1, 134-139.

Speck, O., Ernst, T., Braun, J., Koch, C., Miller, E., \& Chang, L. (2000). Gender differences in the functional organization of the brain for working memory. NeuroReport,11, 25812585.

Systat Software (Version 13.0) [software]. (2008). Retrieved from: http://www.systat.com/. 